\title{
An Unusual Presentation of CLL/SLL on Mammography: Case Report
}

\author{
Anas Mohamed ${ }^{1}$, Ahmed Younes ${ }^{1}$, John Stephen Stalls ${ }^{1}$, Aisha Kousar ${ }^{1}$, and Tian Li $^{1}$ \\ ${ }^{1}$ East Carolina University
}

November 18, 2020

\begin{abstract}
Chronic lymphocytic leukemia/small lymphocytic lymphoma (CLL/SLL) of the breast is rare. We report a 59-year-old female with remote history of ductal carcinoma in situ treated with radiation and tamoxifen, who was recently diagnosed with CLL/SLL of contralateral breast after a localized suspicious cluster of masses was detected on routine mammography.
\end{abstract}

\section{An Unusual Presentation of CLL/SLL on Mammography: Case Report}

Anas Mohamed ${ }^{1,2}{ }^{*}$, Ahmed I. Younes ${ }^{1 *}$, Stephen Stalls ${ }^{1}$, Aisha Kousar ${ }^{1}$, and Tian $\mathrm{Li}^{1}$.

${ }^{1}$ Department of Pathology and Laboratory Medicine, East Carolina University/Vidant Medical Center, Greenville, NC.

${ }^{2}$ Omar Almukhtar University, Faculty of Medicine. Albaida, Libya.

*These two authors should be considered joint first author.

Keywords: CLL/SLL, Breast, Mammography, DCIS, radiation.

\section{Key Clinical Message}

Although rare, breast CLL/SLL should be considered in the differential diagnosis of a breast mass. A high index of suspicion is needed to differentiate this neoplasm from more common breast carcinomas like solid variant of invasive lobular carcinoma.

Correspondence: Anas Mohamed, M.D. Department of Pathology and Laboratory Medicine, East Carolina University/Vidant Medical Center, Greenville, NC.

Email: mohamedan19@ecu.edu

Mobile: +17736291757

ORCID: 0000-0003-0807-5616

\section{Introduction}

To date, five cases have been reported describing the involvement of the breast tissue by CLL/SLL. However, CLL/SLL in those patients was detected as a concurrent tumor with breast carcinoma $(1,2)$ or as an abnormal palpable mass due to sentinel lymph nodes infiltration $(3,4)$. Our patient presented with a unilateral cluster of masses that was restricted to the upper outer quadrant of the right breast. The lesion was neither palpable nor painful, and there were no associated overlying skin changes or axillary lymphadenopathy.

\section{Case History}


A 59-year-old African American woman was referred to our hospital for a suspicious lesion within the right breast discovered by a routine mammography scan. She was diagnosed with intermediate-grade ductal carcinoma in situ (DCIS) of the left breast 13 years before presentation, which was positive for ER, PR, and negative for HER2. She was treated by partial mastectomy, followed by a combined treatment of whole breast radiation and anti-hormonal therapy (tamoxifen). The lesion appeared as a focal asymmetry within the upper outer quadrant of the right breast (Fig. 1A). Targeted ultrasonography was performed and revealed a cluster of multiple prominent lesions, the largest of which was $1 \mathrm{~cm}$ in diameter (Fig. 1B); this finding was categorized as BIRADS 4. Therefore, an ultrasound-guided biopsy was recommended.

The lesion demonstrated solid hypercellular sheets of small dark basophilic cells within a background of benign breast tissue (Fig. 2A and B). No abnormal vascularity was observed. Primary and metastatic breast carcinoma were ruled out by the entirely negative staining pattern within the tumor cells using pancytokeratin (Fig. 2C). A low-grade pattern of KI-67 was seen with higher proliferation centers. While BCL-6 was weakly positive in a subset of the neoplasm, strong nuclear positivity was not observed (Fig. 2D). The aggregates of small neoplastic cells were positive for CD5 (Fig. 2E), PAX-5, BCL-2, CD21, CD23 (Fig. 2F), and CD43, and negative for BCL-1 (Fig. 2G) and CD10 (Fig. 2H). Moreover, fluorescent in situ hybridization (FISH) was negative for CCND1. Three months later, the CT scan demonstrated moderate adenopathy within the lower neck, chest, abdomen, and pelvis. Her CBC showed lymphocytosis, monocytosis, and smudge cells. Other than the left breast DCIS, her past medical history included essential hypertension, morbid obesity, cholecystectomy, and incisional hernia.

\section{Discussion}

Leukemic or lymphoma involvement of the breast could be easily misinterpreted as an invasive carcinoma on imaging due to their overall low incidence or their presentation together with coexisting breast cancer (5). Therefore, the importance of distinguishing between breast lymphoma and carcinoma conditions has recently emerged. Although mammography has been proven to be highly sensitive in detecting breast carcinoma, breast lymphoma cannot be solely diagnosed based on imaging (6). In our case, the lesion appeared as a round or oval mass, which has been observed in the majority of breast lymphoma cases (7). Other less common radiological findings include asymmetry, mammographically detectable lymphedema, and skin thickening (7). In contrast to breast carcinoma, breast lymphoma rarely, if ever, depicts microcalcifications on imaging (7). Therefore, different studies have stated the importance of various imaging modalities, such as MRI, to differentiate invasive breast carcinoma versus other hematologic malignancies of the breast (1).

Microscopic evaluation revealed small, monomorphic, mature lymphocytes with no evidence of normal lymph node architecture, including the capsule. Variable pale proliferation centers were seen where mitotic activity was higher compared to other areas. Ki-67 confirmed the mitotic activity spread. The lymphocytes were positive for CD5, PAX5, CD23, and CD43, which are the most consistent markers for CLL/SLL (8), while they were negative for germinal center markers such as CD10 and BCL6. Although the presence of breast carcinoma in our case was excluded by the negative expression of pan cytokeratin, a high index of suspicion is essential to distinguish between breast CLL/SLL and some histologic subtypes of carcinoma. For instance, solid variant of invasive lobular carcinoma can exhibit small, regular sized, discohesive cells arranged in sheets (9), which can morphologically mimic CLL/SLL. Furthermore, the absence of CCND1 on FISH excluded the majority of mantle cell lymphoma subtypes, which was confirmed with negative BCL1 (10).

Hematologic malignancies of the breast tend to present differently based on their classification. For instance, acute myelogenous leukemia could present as granulocytic myeloid sarcoma (11), while acute lymphocytic leukemia of the breast could present as a diffuse bilateral process with lymphadenopathy (12). Our patient's peripheral blood exhibited lymphocytosis with characteristic smudge cells (basket cells) which is a typical feature of CLL/SLL. However, it was not prominent upon her presentation, and the lymphocytes began to rise one month after the biopsy was obtained.

Our patient previously received whole breast irradiation with a total dose of 5040 cGy; previous reports showed that radiation might be absorbed in the contralateral breast from the scattered external beam (13). 
Physical DNA damage caused by radiation can activate tumor suppressor p53, which was proven to cause radiation-induced lymphoma (14). Hence, we hypothesize that there might be a connection between her CLL/SLL diagnosis and her past medical history, which would need further research to confirm.

Finally, it is essential to consider that CLL/SLL can present as unilateral irregular breast aggregates on mammography. It is an uncommon entity that should be further studied, particularly in patients with a history of DCIS or those who receive radiation treatment even if it is localized. Her CLL/SLL was discovered on imaging before she developed any systemic manifestations, which is also uncommon.

\section{Conclusion}

In conclusion, our patient developed CLL/SLL, which was confined to the right breast and was not associated with any breast carcinoma. Awareness of this patient's profile, particularly the long duration between DCIS treatment and the development of CLL/SLL, could give insight into the value of long-term surveillance for such patients after recovery. It also highlights the role of imaging techniques and pathological evaluation in recognition of breast leukemia and lymphoma.

\section{Author Contribution}

Anas Mohamed \& Ahmed I Younes: Drafted and wrote the article and designed figures.

Stephen Stalls: Edited and reviewed the article.

Aisha Kousar: Edited and reviewed the article.

Tian Li: Revised the manuscript critically and provided final suggestions for final preparation.

\section{Conflicts of Interest:}

All authors declare no conflict of interest.

\section{Ethical Approval:}

This manuscript is in compliance with the IRB policy of East Carolina University Department of Pathology and Laboratory Medicine.

\section{Funding:}

The article processing charges for this manuscript were provided by the East Carolina University Department of Pathology and Laboratory Medicine.

\section{Acknowledgements:}

The authors thank Dr. John Fallon, chair of the East Carolina University Department of Pathology and Laboratory Medicine, for his support for this manuscript including securing funding for publication.

\section{References}

1. Dialani V, Mani K, Johnson NB. Chronic lymphocytic leukemia involving the breast parenchyma, mimicker of invasive breast cancer: differentiation on breast MRI. Case Rep Med. 2013;2013:603614. doi:10.1155/2013/603614.

2. Zhong J, Di L, Zheng W. Synchronous breast cancer and breast lymphoma: two case reports and literature review. Chin J Cancer Res. 2014;26(3):355-359. doi:10.3978/j.issn.1000-9604.2014.06.04.

3. Cheung KJ, Tam W, Chuang E, Osborne MP. Concurrent invasive ductal carcinoma and chronic lymphocytic leukemia manifesting as a collision tumor in breast. Breast J. 2007;13(4):413-417. doi:10.1111/j.1524-4741.2007.00451.x.

4. Sheen-Chen SM, Eng HL. Development of malignant lymphoma subsequent to breast cancer. Eur J Cancer Care (Engl). 2007;16(4):331-332. doi:10.1111/j.1365-2354.2006.00750.x.

5. Surov A, Wienke A, Abbas J. Breast leukemia: an update. Acta Radiol. 2012;53(3):261-266. doi:10.1258/ar.2011.110470. 
6. Zeeshan M, Salam B, Khalid QSB, Alam S, Sayani R. Diagnostic Accuracy of Digital Mammography in the Detection of Breast Cancer. Cureus. 2018;10(4):e2448. Published 2018 Apr 8. doi:10.7759/cureus.2448.

7. Raj SD, Shurafa M, Shah Z, Raj KM, Fishman MDC, Dialani VM. Primary and Secondary Breast Lymphoma: Clinical, Pathologic, and Multimodality Imaging Review. Radiographics. 2019;39(3):610625. doi:10.1148/rg.2019180097.

8. Wang HY, Zu Y. Diagnostic Algorithm of Common Mature B-Cell Lymphomas by Immunohistochemistry. Arch Pathol Lab Med. 2017;141(9):1236-1246. doi:10.5858/arpa.2016-0521-RA.

9. McCart Reed AE, Kutasovic JR, Lakhani SR, Simpson PT. Invasive lobular carcinoma of the breast: morphology, biomarkers and 'omics. Breast Cancer Res. 2015;17(1):12. Published 2015 Jan 30. doi:10.1186/s13058-015-0519-x

10. Ott MM, Helbing A, Ott G, et al. bcl-1 rearrangement and cyclin D1 protein expression in mantle cell lymphoma. J Pathol. 1996;179(3):238-242. doi:10.1002/(SICI)1096-9896(199607)179:3<238::AIDPATH566>3.0.CO;2-W.

11. Barloon TJ, Young DC, Bass SH. Multicentric granulocytic sarcoma (chloroma) of the breast: mammographic findings. AJR Am J Roentgenol. 1993;161(5):963-964. doi:10.2214/ajr.161.5.8273636.

12. Besina S, Rasool Z, Samoon N, Akhtar OS. Acute lymphoblastic leukemia presenting as a breast lump: A report of two cases. J Cytol. 2013;30(3):201-203. doi:10.4103/0970-9371.117644.

13. Hooning MJ, Aleman BM, Hauptmann M, et al. Roles of radiotherapy and chemotherapy in the development of contralateral breast cancer. J Clin Oncol. 2008;26(34):5561-5568. doi:10.1200/JCO.2007.16.0192.

14. Lee CL, Castle KD, Moding EJ, et al. Acute DNA damage activates the tumour suppressor p53 to promote radiation-induced lymphoma. Nat Commun. 2015;6:8477. Published 2015 Sep 24. doi:10.1038/ncomms9477.

\section{Figure Legends}

Figure 1. (A) Cluster of radiopaque masses confined to superior lateral quadrant of the right breast. (B) Ultrasonography image of the hypoechoic area that revealed the diagnosis of CLL/SLL.

Figure 2. (A) Hematoxylin and eosin (H\&E) staining shows breast tissue replacement by solid sheets of tumor cells with a background of adipose tissue (40x). (B) Small dark round lymphocytes on H\&E (400x). (C) Tumor cells exhibit negative staining with pan-cytokeratin (200x). (D) Lack of strong nuclear positivity for BCL6 (200x). (E) Neoplastic cells are positive for CD5 (200x). (F) Neoplastic cells are positive for CD23 (200x). (G) Tumor cells exhibit negative staining for BCL1 (200x). (H) CD10 is negative in neoplastic cells (200x). 
Figure 1

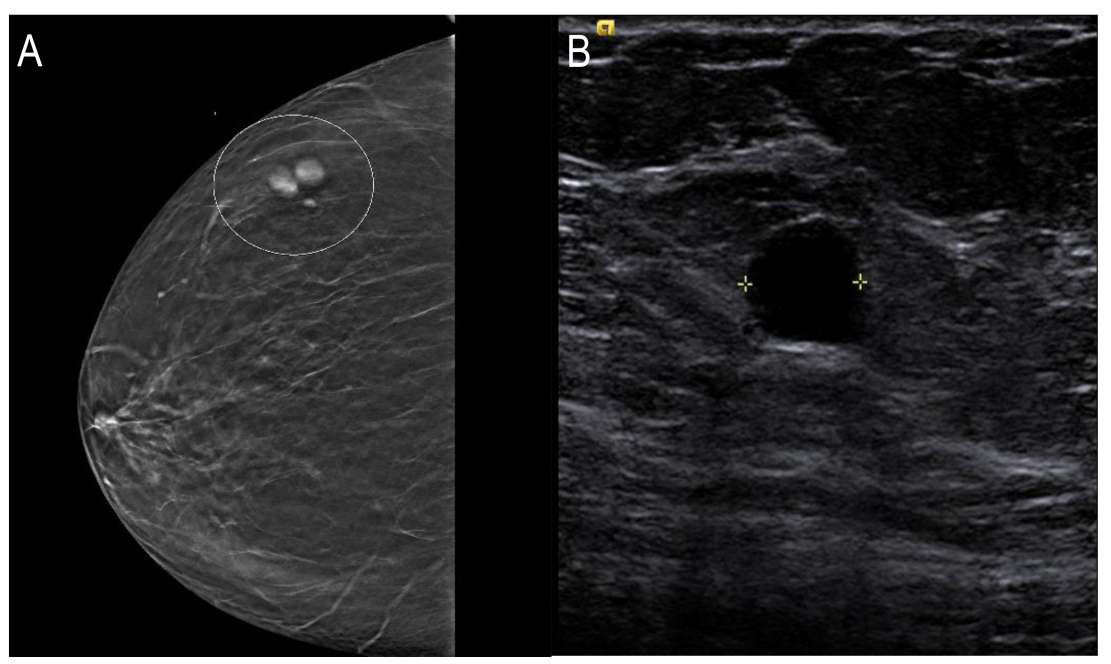

Figure 2

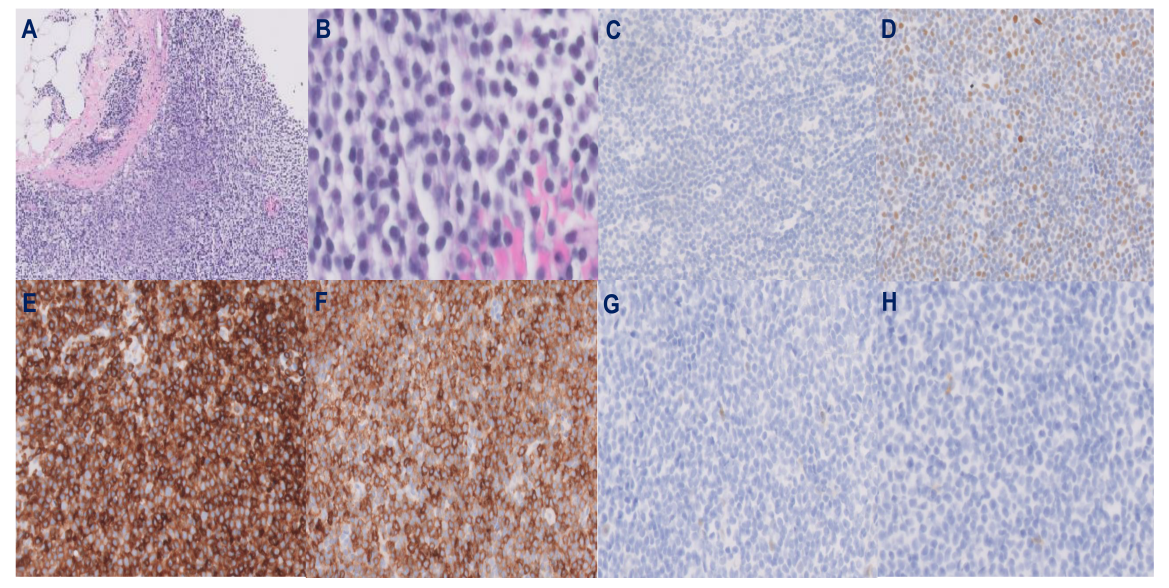

